\title{
Precession Motion in Levitated Optomechanics
}

\author{
Muddassar Rashid, ${ }^{*}$ Marko Toroš, ${ }^{\dagger}$ Ashley Setter, and Hendrik Ulbricht ${ }^{\ddagger}$ \\ Department of Physics and Astronomy, University of Southampton, Southampton, SO17 1BJ, United Kingdom
}

(Received 21 May 2018; published 17 December 2018)

\begin{abstract}
We investigate experimentally the dynamics of a nonspherical levitated nanoparticle in a vacuum. In addition to translation and rotation motion, we observe the light torque-induced precession and nutation of the trapped particle. We provide a theoretical model, which we numerically simulate and from which we derive approximate expressions for the motional frequencies. Both the simulation and approximate expressions we find in good agreement with experiments. We measure a torque of $1.9 \pm 0.5 \times 10^{-23} \mathrm{~N} \mathrm{~m}$ at $1 \times 10^{-1}$ mbar, with an estimated torque sensitivity of $3.6 \pm 1.1 \times 10^{-31} \mathrm{~N} \mathrm{~m} / \sqrt{\mathrm{Hz}}$ at $1 \times 10^{-7} \mathrm{mbar}$.

DOI: 10.1103/PhysRevLett.121.253601
\end{abstract}

Introduction.-A typically levitated optomechanical setup comprises of a particle, which is trapped using a tightly focused laser beam. The trapped particle is best described as a harmonic oscillator and can exhibit a rich variety of dynamics. For example, the dynamics can be underdamped or overdamped depending on the number of collisions controlled by the background gas pressure. The dynamics can be linear or can show strong nonlinearities depending on the oscillation amplitude, which is controlled by temporal and spatial modulated external electric, magnetic, and light forces: The dynamics can be driven or cooled. This tunability of the dynamics has enabled various studies of the rich physics of this harmonic oscillator, among them Brownian motion [1], nonlinear dynamics [2,3], test of fluctuation theorems and nonequilibrium physics [4-6], and thermodynamics in the single-particle regime [7-9]. The unique properties of the underlying dynamics and the ability to control levitated optomechanics demonstrate its immense potential for sensing [10-12] as well as to address fundamental questions in physics [13-15].

The center-of-mass (c.m.) translation motion of the trapped nanoparticle has been studied in quite some detail already. Optical feedback, cavity-assisted schemes, and electric forces have been used to control the translation and have been ultimately used to cool the motion to millikelvin temperatures [16-19] and below [20], already close to the ground state of the harmonic oscillator.

In addition to exhibiting translation motion, trapped particles can also show rotation [21-23] and libration [24] motion. Different light-matter physics has been used to drive and control the rotation of levitated particles, such as the polarizability anisotropy in silicon rods coupling to the polarization of light [25]. Rotational frequencies of up to some gigahertz $[26,27]$ have been observed, limited only by the centrifugal damage threshold of the rotating particle.

Variation of the linear to circular polarization of light gives a handle to switch between rotation and libration.
Libration has been demonstrated experimentally with trapped nanodiamonds [24], silicon rods [25], and dumbbells [27]. Like translation, libration motion is described by a harmonic oscillator model and is therefore a candidate to apply similar optical techniques for cooling with reasonable promise to reach a quantum ground state, making libration a stark contender in the race towards the quantum regime. First proposals discuss the usefulness of libration to generate macroscopic quantum states such as angular superpositions [28,29]. Additionally, both rotation and libration motion promise unprecedented high levels of sensitivity $[24,30,31]$ for the detection of weak forces such as gravity [28,32] and dispersive forces [33].

In this Letter, we report on the observation of lightinduced precession motion of a nonspherical silica particle compound. We give a theoretical description of the system and numerically simulate the model. We identify the mechanical frequencies in the experimental spectrum: in particular, translation, rotation (spin), precession, and nutation motion. We investigate the precession by variation of the background gas pressure and of the power of the trapping laser in agreement with the theoretical model. In addition, we discuss the possibilities for torque sensing applications.

Theoretical model.-We consider an anisotropic polarizable particle, which is optically trapped by an elliptically polarized Gaussian laser beam. Part of the scattered photons are collected and directed, using optical elements, towards a single photodetector. The scattered light is mixed with a local oscillator to obtain the direct homodyne photocurrent $I_{\text {exp }}$; see Fig. 1(a) [34]. This experimental situation has been analyzed theoretically using a quantum model [35], as well as numerically, using an approximate classical model [36]. We now summarize the dynamics referring to the latter, where we assume that we are at a relatively high pressure such that we can neglect photonrecoil heating terms. 
(a)

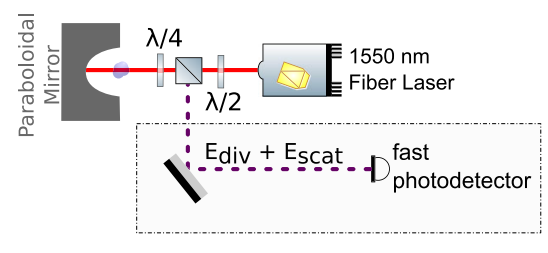

(b)

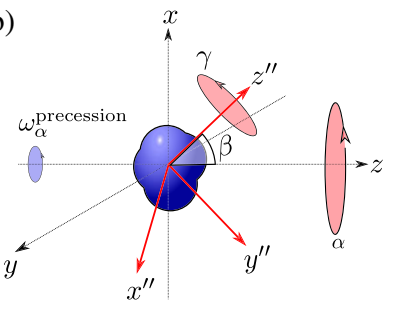

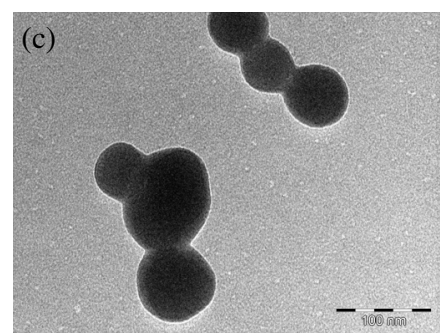

FIG. 1. The levitated optomechanical system: (a) A $1550 \mathrm{~nm}$ laser beam is focused by the paraboloidal mirror, and the particle is trapped in the focus. Once trapped, the scattered light $E_{\text {scat }}$ from the particle is collected and directed towards the detection system. The interference between the scattered and the diverging electric fields, $E_{\mathrm{div}}$, is used to detect the motion of the particle. (b) Parametrization: A nonspherical particle that is trapped by a laser field propagating in the $z$ direction. The laboratory axes are denoted by $x, y$, and $z$, while the body-frame axes are denoted by the $x^{\prime \prime}, y^{\prime \prime}$, and $z^{\prime \prime}$. The relation between the two frames is parametrized by the Euler angles $\alpha, \beta$, and $\gamma$ in the $z-y^{\prime}-z^{\prime \prime}$ convention. $\alpha$ denotes the angle of rotation about the laboratory $z$ axis (from $x$ towards $y$ ). $\beta$ is the angle between the laboratory $z$ axis and the body $z^{\prime \prime}$ axis (rotated about the $y^{\prime}$ axis, i.e., the $y$ axis after it has been rotated by $\alpha$ about the $z$ axis; from $z$ towards $x$ ). $\gamma$ denotes the angle of rotation about the body frame $z^{\prime \prime}$ axis (from $x^{\prime \prime}$ towards $y^{\prime \prime}$ ). (c) TEM image of compound silica particle solution in water, a few weeks after preparation. The original particle solution is made of $50 \mathrm{~nm}$ radius silica spheres (Corpuscular Inc.).

The dynamics can be described in a 12-dimensional phase space of the following classical variables: center-ofmass position $\boldsymbol{r}=(x, y, z)^{\top}$, center-of-mass conjugate momentum $\boldsymbol{p}=\left(p_{x}, p_{y}, p_{z}\right)^{\top}$, angle $\boldsymbol{\phi}=(\alpha, \beta, \gamma)^{\top}$, and angle conjugate momentum $\pi=\left(\pi_{\alpha}, \pi_{\beta}, \pi_{\gamma}\right)^{\top}$, where the angles are defined in the Euler $z-y^{\prime}-z^{\prime \prime}$ convention [see Fig. 1(b)]. In particular, the dynamics is given by (in Itó form)

$$
\begin{aligned}
& d \boldsymbol{r}=-\partial_{\boldsymbol{p}} H_{\text {free }} d t, \\
& d \boldsymbol{p}=-\partial_{\boldsymbol{r}} H_{\text {grad }} d t+d \boldsymbol{p}_{\text {scatt }}+d \boldsymbol{p}_{\text {coll }}, \\
& d \boldsymbol{\phi}=\partial_{\boldsymbol{\pi}}\left(H_{\text {free }}+H_{\text {grad }}\right) d t, \\
& d \boldsymbol{\pi}=-\partial_{\boldsymbol{\phi}}\left(H_{\text {free }}+H_{\text {grad }}\right) d t+d \boldsymbol{\pi}_{\text {scatt }}+d \boldsymbol{\pi}_{\text {coll }},
\end{aligned}
$$

where $H_{\text {free }}$ and $H_{\text {grad }}$ denote the free Hamiltonian and the gradient potential, respectively, $d \boldsymbol{p}_{\text {scatt }}$ and $d \boldsymbol{\pi}_{\text {scatt }}$ denote the nonconservative terms induced by photon scattering, and $d \boldsymbol{p}_{\text {coll }}$ and $d \boldsymbol{\pi}_{\text {coll }}$ denote the nonconservative terms, which arise from gas collisions. Specifically, $d \boldsymbol{p}_{\text {scatt }}$ corresponds to the radiation pressure scattering force, which displaces the particle along the positive $z$ direction, and $d \boldsymbol{p}_{\text {coll }}$ denotes the terms which tend to thermalize the center-of-mass motion to the temperature $T$ of the gas of particles. Similarly, $d \boldsymbol{\pi}_{\text {scatt }}$ denotes the terms that quantify the transfer of angular momentum from the photons to the particle, i.e., the driving terms, and $d \boldsymbol{\pi}_{\text {coll }}$ denotes the terms that tend to thermalize rotations to the temperature of the gas, i.e., the friction and diffusive terms (see Supplemental S1 [37]).

We consider a specific experimental situation, where we illustrate the physical content of Eqs. (1)-(4), and we obtain an approximate expression for the dominant mechanical frequencies. Specifically, we consider the experimental situation that produces the power spectral density in Fig. 2, where the rotational frequencies are significantly higher or lower than the translational ones. To obtain the dominant mechanical frequencies, we can in a first approximation treat translation and rotation as decoupled motion.

We start by looking at translational degrees of freedom (d.o.f.). We suppose that $|x(\boldsymbol{r} / \lambda)| \ll 1$, where $\lambda$ is the laser wavelength, which limits translations to harmonic oscillations. In particular, the frequencies for the $x, y, z$ motion are given by

$\omega_{x}^{2}=\frac{2 P a_{1} \chi_{0}}{c \sigma_{L} w_{0}^{2} \rho}, \quad \omega_{y}^{2}=\frac{2 P a_{2} \chi_{0}}{c \sigma_{L} w_{0}^{2} \rho}, \quad \omega_{z}^{2}=\frac{2 P \chi_{0}}{c \sigma_{L} \rho z_{R}^{2}}$,

respectively, where $P$ is the laser power, $\sigma_{L}=\pi w_{0}^{2}$ is the effective laser beam cross section area, $w_{0}$ is the mean beam waist radius, $a_{1}$ and $a_{2}$ quantify the asymmetry of the beam along the $x$ and $y$ directions, respectively, $z_{R}$ is the Rayleigh length, $\rho$ is the particle density, $\chi_{0}=\frac{1}{3} \sum_{i=1}^{3} \chi_{i}$ is an effective susceptibility of the particle, and $c$ is the speed of light. These frequencies are obtained directly from $H_{\text {grad }}$ by expanding to the order of $\mathcal{O}\left((\mathbf{r} / \lambda)^{2}\right)$.

The rotational frequencies arise from (i) the transfer of angular momentum during photon scattering and (ii) the gradient torque. On one hand, the scattering torque drives the system into a fast spinning motion, while, on the other hand, the gradient torque tends to align the system with the polarization of the incoming beam in such a way to minimize the electric dipole potential energy, resulting in nutation and precession. We now give an intuitive picture of the two mechanisms and discuss the resulting rotational motions (for further information, see Supplemental S2 [37]).

The mechanism (i) can be understood in terms of the angular momentum carried by the incoming light beam (in a particle picture, one can think of an individual photon carrying a small amount of angular momentum, e.g., $\hbar$ for circular polarization). During scattering, the angular momentum is transferred to the nanoparticle, where the 


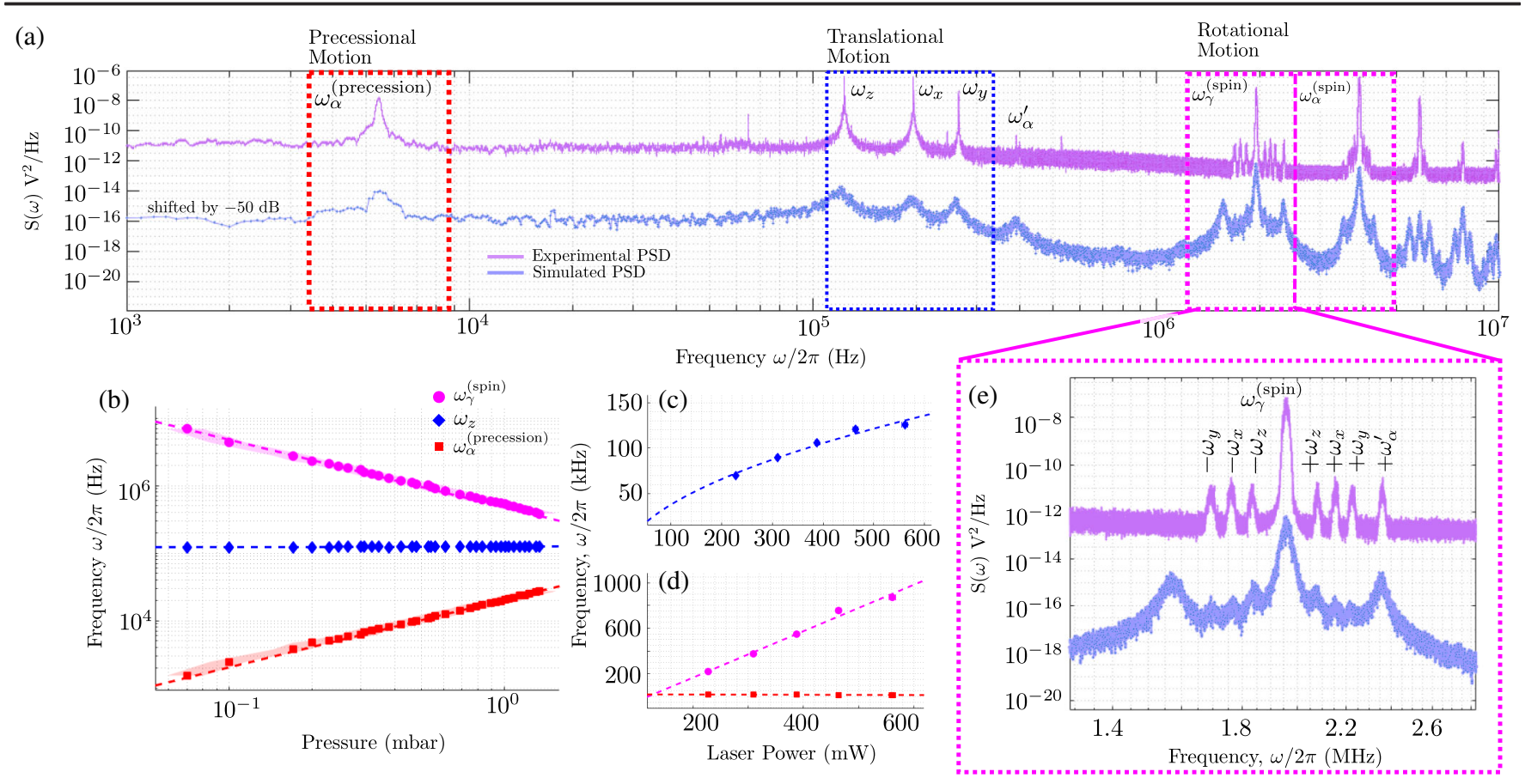

FIG. 2. Measured spectrum: (a) the full experimental spectrum obtained at $1 \times 10^{-1} \mathrm{mbar}$ (shown in purple). It includes contributions from translation, rotation, and precession motion. Additionally, a simulated spectrum (shown in blue) fitted to the experimental PSD is shifted for visibility. The simulated spectrum is obtained with $10^{8}$ time steps. (b) shows the frequency dependencies with pressure for translation (blue), rotation (magenta), and precession (red). The inverse relation is a signature of rotation, while the direct proportionality is signature of precession. (c) shows the change in the translation frequency due to the square root of laser power. (d) shows the linear dependency of rotation, $\omega_{\gamma}^{\text {(spin) }}$, with laser power, while precession is independent of laser power. (e) Detailed spectrum of rotation frequency $\omega_{\gamma}^{(\text {spin) }}$ with translation sidebands and $\omega_{\alpha}^{\prime}$ an additional frequency peak appearing in the motion of $\alpha$ motion.

amount that is transferred depends on the susceptibility anisotropy and orientation of the nanoparticle. As a consequence, the particle starts to spin, until an asymptotic rotational frequency is reached, which is constrained by friction due to gas collisions. In particular, we consider the experimental situation where the photon scattering gives rise to the high spinning frequencies:

$$
\begin{aligned}
& \omega_{\alpha}^{\text {(spin) }}=\frac{\Gamma_{s}}{\Gamma_{c}} \mathcal{F}_{\alpha}^{\text {(spin) }}\left(\chi, I, \beta_{0}\right), \\
& \omega_{\gamma}^{\text {(spin) }}=\frac{\Gamma_{s}}{\Gamma_{c}} \mathcal{F}_{\gamma}^{\text {(spin) }}\left(\chi, I, \beta_{0}\right),
\end{aligned}
$$

about the $z$ and $z^{\prime \prime}$ axis, respectively [see Eqs. (S18) and (S19) [37]]. $\Gamma_{s}$ is the photon scattering rate, $\Gamma_{c}$ is the gas collision rate, and $\mathcal{F}_{\alpha}^{\text {(spin) }}$ and $\mathcal{F}_{\gamma}^{\text {(spin) }}$ are functions of susceptibility tensor $\chi$, moment of inertia tensor $I$, and the equilibrium angle $\beta_{0}$ for the $\beta$ d.o.f., which we now discuss below.

Besides the dominant spinning motions, there are also two additional secondary motions, which arise as a consequence of mechanism (ii). In a nutshell, the gradient torque would like to align the nanoparticle in such a way to minimize the electric dipole potential energy, i.e., $\beta_{0}=(\pi / 2)$, but once the nanoparticle starts to spini.e., it acquires a large angular momenta along the $z$ and $z^{\prime \prime}$ axis - it is unable to fully align but rather settles around an equilibrium position $\beta_{0} \neq(\pi / 2)$, which can be readily understood in terms of angular momenta addition. Any small perturbation, e.g., gas collisions, will make the $\beta$ angle oscillate around the $\beta_{0}$ angle, which results in libration (nutation) motion with frequency

$$
\omega_{\beta}^{\text {(nutation) }}=\frac{\Gamma_{s}}{\Gamma_{c}} \mathcal{F}_{\beta}^{(\text {nutation })}\left(\chi, I, \beta_{0}\right),
$$

where $\Gamma_{s}$ is the scattering rate, $\Gamma_{c}$ is the gas collision rate, and $\mathcal{F}_{\beta}^{(\text {nutation })}$ is a function of the susceptibility tensor $\chi$, the moment of inertia tensor $I$, and the equilibrium angle $\beta_{0}$ [see Supplemental Eq. (S20) [37]].

In addition, the coupling between $\beta$ and $\alpha$ also creates a second frequency for the $\alpha$ motion, which is given by

$$
\omega_{\alpha}^{(\text {precession })}=\omega_{\alpha}^{(\text {spin })}-\omega_{\beta}^{(\text {nutation })} .
$$

This precession motion can be seen as a consequence of the $\beta$ motion, which perturbs back the $\alpha$ motion, and can be visualized as a slow precession of the $z^{\prime \prime}$ axis about the $z$ axis [see Fig. 1(b)]. The $\alpha$ d.o.f. has thus two distinct motions: a fast spinning motion with the frequency given in 
Eq. (6) and a slow precession motion with the frequency given in Eq. (9).

From Eqs. (6)-(8), noting that $\left(\Gamma_{s} / \Gamma_{c}\right) \propto(P / p)$, we find that $\omega_{\alpha}^{\text {(spin) }}, \omega_{\gamma}^{\text {(spin) }}$, and $\omega_{\beta}^{\text {(nutation) }}$ scale linearly with the laser power $P$ and are inversely proportional to the gas pressure $p$, where we have assumed that the equilibrium position $\beta_{0}$ does not change significantly near an initially chosen power $P_{0}$ and pressure $p_{0}$. On the other hand, the precession frequency $\omega_{\alpha}^{\text {(precession) }}$ given in Eq. (9) can scale differently depending on the values of $\left.\partial_{P} \Delta \omega\right|_{P_{0}}$ and $\left.\partial_{p} \Delta \omega\right|_{p_{0}}$, where $\Delta \omega=\omega_{\alpha}^{\text {(spin) }}-\omega_{\beta}^{\text {(nutation) }}$.

Experiments.-The optical trap is shown in Fig. 1(a). In the experiments presented, we use initially individual silica nanoparticles dispersed in water. We observe ageing of the solution with the result of clustering of the nanospheres into compounds of two to three nanospheres, a few weeks after preparation, as shown in Fig. 1(c). The aged solution is delivered to the trap using a nebulizer. The optical scattering force limits the maximum particle mean radius that can be optically trapped to about $150-200 \mathrm{~nm}$.

Results.-The power spectral density (PSD) shown in Fig. 2(a) is generated from the time trace recorded as the photodetector signal $I_{\text {exp }}$ over a time interval of one second. The PSD shows a rich spectrum of frequencies, which respond differently for changing laser power and background gas pressure.

Translation motion is observed at frequencies $\omega_{x}=$ $2 \pi \times 196 \mathrm{kHz}, \omega_{y}=2 \pi \times 246 \mathrm{kHz}$, and $\omega_{z}=2 \pi \times 124 \mathrm{kHz}$ in Fig. 2(a). We do not observe pressure dependency of the translation frequencies [see Fig. 2(b)] but find that they scale proportionately to the square root of laser power, $P$, in agreement with Eq. (5); see Fig. 2(c). The $x$ and $y$ peaks are separated, because we use elliptically polarized light, which after it is reflected from the paraboloidal mirror generates an asymmetric optical trap. The polarization of light was kept constant during the course of the experiments in this Letter.

From the experimental data, we find the fundamental frequencies for the rotational motions $\omega_{\gamma}^{\text {(spin) }}$ and $\omega_{\alpha}^{\text {(spin) }}$ to be $2 \pi \times 1.9$ and $2 \pi \times 3.8 \mathrm{MHz}$, respectively, which are reproduced using numerical simulations and the approximate Eqs. (6) and (7). The rotational frequency $\omega_{\gamma}^{\text {(spin) }}$ changes with the damping $\Gamma_{c}$, which depends linearly on the gas pressure $p$, i.e., $\omega_{\gamma}^{\text {(spin) }} \propto\left(1 / \Gamma_{c}\right) \propto(1 / p)$. This is a clear signature of rotation motion, as shown in Fig. 2(b). We also observe a dependency of the frequency on the laser power $P$, as shown in Fig. 2(d), in agreement with the dependency on the photon scattering rate, $\Gamma_{s} \propto P$. Zooming to the fundamental frequency $\omega_{\gamma}^{(\text {spin })}$ reveals sidebands [see Fig. 2(e)], which are the addition and subtraction of the three translational frequencies. Using the numerical simulation, we identify another mode in $\alpha$ rotation, with frequency $\omega_{\alpha}^{\prime}=2 \pi \times 393 \mathrm{kHz}$ : This gives rise to the sideband in $\omega_{\gamma}^{(\text {spin })}$. In the presented data set, we resolve only one of the $\omega_{\alpha}^{\prime}$ sideband peaks (for further information, see Supplemental S4 [37]). The light-matter interactions introduce couplings between translation and rotation, which explain the observed sidebands and higher harmonics in agreement with numerical simulation (see Supplemental S1 and S3 [37] for further details). We also observe $\omega_{\alpha}^{\text {(spin) }}$ to scale linearly with power and inversely with pressure.

Further to observing translation and rotation peaks, we observe a frequency at $2 \pi \times 5.4 \mathrm{kHz}$, as shown in Fig. 2(a). This frequency is well isolated and characterized by its dependency on laser power $P$ and gas pressure $p$. We associate this low frequency with the gradient torqueinduced precession $\omega_{\alpha}^{\text {(precession) }}$. From Eq. (9), Taylor expanding about the initial pressure $p_{0}$, we find that the dominant term is linear in $p$ (the constant terms cancel), i.e., $\omega_{\alpha}^{\text {(precession) }}=\left.\partial_{p}(\Delta \omega)\right|_{p_{0}} p$. On the other hand, we find a weak dependence on the laser power $P$, i.e., $\omega_{\alpha}^{\text {(precession) }}=\left.\Delta \omega\right|_{P_{0}}$, where $P_{0}$ is the initial laser power. We verify these frequency dependencies on gas pressure and laser power in Figs. 2(b) and 2(d), respectively. We exclude $\omega_{\alpha}^{\text {(precession) }}$ to be caused by nonlinear translational motion, as the translation frequencies do not change with pressure. This is evident from Fig. 2(b), where $\omega_{z}$ is constant. Thus, we conclude that the observed frequency and its behavior are signatures of precession motion as described by Eq. (9).

The precession motion arises due to the fast spinning $\alpha$ d.o.f. as well as the nutation motion of $\beta$. The observation of precession is thus also an indirect indication of nutation motion. Using numerical simulations, we find that $\beta$ rotation to be exhibiting libration motion, which is linearly dependant on power and inverse proportional to gas pressure, as a result of coupling between $\beta$ and $\alpha$ d.o.f.

Discussion.-From the theoretical analysis, the precession motion arises due to an optical torque acting upon the trapped particle. Torque can also be generated by an external force, which opens the way for the sensitive detection of forces by precession. In particular, by combining Eqs. (6)-(9), we get an expression for the $\alpha$ component of the photon scattering torque:

$$
N_{\alpha}=\Gamma_{c} \sin ^{2}\left(\beta_{0}\right)\left(\frac{2 I_{1} I_{2}\left(I_{1}+I_{2}\right)}{I_{1}^{2}+I_{2}^{2}}\right) \omega_{\alpha}^{\text {(precession })},
$$

where we have kept only the dominant terms, denote the term in the large parentheses by $\mathcal{J}$, and name it the effective moment of inertia. Using the experimentally measured $\omega_{\alpha}^{\text {(precession) }}$ and estimating $\mathcal{J}$, we achieve a measured torque of $N_{\alpha}=1.9 \pm 0.5 \times 10^{-23} \mathrm{Nm}$ at $1 \times 10^{-1} \mathrm{mbar}$, in comparison to the measurement of nanoscale torque sensors reported down to $10^{-20} \mathrm{Nm}$ [42] and to estimates of $10^{-22} \mathrm{Nm}$ [30] for silicon nanorods. 
We now consider an additional small external torque acting on $\alpha$, which we denote by $\delta N_{\text {ext }}$. We denote the change in the precession frequency sensitivity by $\delta \omega_{\alpha}$ (see Supplemental S5 [37]), and, assuming that the equilibrium value of $\beta_{0}$ remains largely unaffected, we obtain the torque sensitivity $\delta N_{\text {ext }}=\Gamma_{c} \sin \left(\beta_{0}\right)^{2} \mathcal{J} \delta \omega_{\alpha}$. Using the current experimental parameters, i.e., moments of inertia and the precessional frequency, we estimate a torque sensitivity of $3.6 \pm 1.1 \times 10^{-31} \mathrm{Nm} / \sqrt{\mathrm{Hz}}$ at $1 \times 10^{-7} \mathrm{mbar}$, at which point photon shot noise becomes a dominant source of noise (see Supplemental S7 [37]). This torque sensitivity is in comparison to the values obtained using libration motion, which is at $1 \times 10^{-29} \mathrm{Nm} / \sqrt{\mathrm{Hz}}$ at $10^{-9}$ mbar [24] (for further discussion, see Supplemental S6 and S8 [37]).

Conclusions. - We have observed precession motion in levitated optomechanics and inferred the presence of nutation motion. We present a theory of this motion and show that it arises from the equations of motion for a rotating object experiencing scattering and gradient forces and torques. Additionally, we characterize the rich spectrum containing translations, rotations, and higher harmonics. We further show a measured torque of $10^{-23} \mathrm{~N} \mathrm{~m}$ at $10^{-1}$ mbar and predict the ability to reach sensitivities down to $10^{-31} \mathrm{~N} \mathrm{~m} / \sqrt{\mathrm{Hz}}$ at $10^{-7}$ mbar.

Thus, precession motion is a d.o.f. that could be utilized for torque sensing with the sensitivities to resolve singleelectron [43] and even nuclear spins [24] at low pressure. This work paves the way for gyroscope applications, as shown in Ref. [44]. The precession motion can also be used for dynamical model selection to distinguish between quantum and classical evolution due to the inherent nonlinearities in rotation motion [45], if sufficient coherence can be prepared. Additionally, the experimental spectrum combined with numerical simulations can be used for shape tomography.

All data supporting this study are openly available from the University of Southampton by following the link in Ref. [46].

We thank C. Timberlake and G. Winstone for discussion and M. Rademacher for assistance with the TEM image. We thank for funding the Leverhulme Trust [RPG-2016046], the EU Horizon 2020 research and innovation program under Grant Agreement No. 766900 [TEQ] and the EU COST action CA15220 (QTSpace). A.S. is supported by the United Kingdom Engineering and Physical Sciences Research Council (EPSRC) under Centre for Doctoral Training Grant No. EP/L015382/1.

*m.rashid@soton.ac.uk

†m.toros@soton.ac.uk

"h.ulbricht@soton.ac.uk

[1] T. Li, S. Kheifets, D. Medellin, and M. G. Raizen, Science 328, 1673 (2010).
[2] J. Gieseler, L. Novotny, and R. Quidant, Nat. Phys. 9, 806 (2013).

[3] P. Z. G. Fonseca, E. B. Aranas, J. Millen, T. S. Monteiro, and P. F. Barker, Phys. Rev. Lett. 117, 173602 (2016).

[4] J. Gieseler, R. Quidant, C. Dellago, and L. Novotny, Nat. Nanotechnol. 9, 358 (2014).

[5] J. Gieseler, M. Spasenović, L. Novotny, and R. Quidant, Phys. Rev. Lett. 112, 103603 (2014).

[6] T. M. Hoang, R. Pan, J. Ahn, J. Bang, H. T. Quan, and T. Li, Phys. Rev. Lett. 120, 080602 (2018).

[7] J. Millen, T. Deesuwan, P. Barker, and J. Anders, Nat. Nanotechnol. 9, 425 (2014).

[8] L. Rondin, J. Gieseler, F. Ricci, R. Quidant, C. Dellago, and L. Novotny, Nat. Nanotechnol. 12, 1130 (2017).

[9] E. B. Aranas, P. Z. Fonseca, P. F. Barker, and T. S. Monteiro, J. Opt. 19, 034003 (2017).

[10] G. Ranjit, M. Cunningham, K. Casey, and A. A. Geraci, Phys. Rev. A 93, 053801 (2016).

[11] E. Hebestreit, M. Frimmer, R. Reimann, and L. Novotny, Phys. Rev. Lett. 121, 063602 (2018).

[12] D. Hempston, J. Vovrosh, M. Toroš, G. Winstone, M. Rashid, and H. Ulbricht, Appl. Phys. Lett. 111, 133111 (2017).

[13] O. Romero-Isart, A. C. Pflanzer, F. Blaser, R. Kaltenbaek, N. Kiesel, M. Aspelmeyer, and J. I. Cirac, Phys. Rev. Lett. 107, 020405 (2011).

[14] A. Bassi, K. Lochan, S. Satin, T. P. Singh, and H. Ulbricht, Rev. Mod. Phys. 85, 471 (2013).

[15] J. Bateman, S. Nimmrichter, K. Hornberger, and $H$. Ulbricht, Nat. Commun. 5, 4788 (2014).

[16] T. Li, S. Kheifets, and M. G. Raizen, Nat. Phys. 7, 18 (2011).

[17] N. Kiesel, F. Blaser, U. Delić, D. Grass, R. Kaltenbaek, and M. Aspelmeyer, Proc. Natl. Acad. Sci. U.S.A. 110, 14180 (2013).

[18] J. Vovrosh, M. Rashid, D. Hempston, J. Bateman, M. Paternostro, and H. Ulbricht, J. Opt. Soc. Am. B 34, 1421 (2017).

[19] A. Setter, M. Toroš, J. F. Ralph, and H. Ulbricht, Phys. Rev. A 97, 033822 (2018).

[20] V. Jain, J. Gieseler, C. Moritz, C. Dellago, R. Quidant, and L. Novotny, Phys. Rev. Lett. 116, 243601 (2016).

[21] Y. Arita, M. Mazilu, and K. Dholakia, Nat. Commun. 4, 2374 (2013).

[22] S. Kuhn, P. Asenbaum, A. Kosloff, M. Sclafani, B. A. Stickler, S. Nimmrichter, K. Hornberger, O. Cheshnovsky, F. Patolsky, and M. Arndt, Nano Lett. 15, 5604 (2015).

[23] A. T. M. A. Rahman and P. F. Barker, Nat. Photonics 11, 634 (2017).

[24] T. M. Hoang, Y. Ma, J. Ahn, J. Bang, F. Robicheaux, Z.-Q. Yin, and T. Li, Phys. Rev. Lett. 117, 123604 (2016).

[25] S. Kuhn, A. Kosloff, B. A. Stickler, F. Patolsky, K. Hornberger, M. Arndt, and J. Millen, Optica 4, 356 (2017).

[26] R. Reimann, M. Doderer, E. Hebestreit, R. Diehl, M. Frimmer, D. Windey, F. Tebbenjohanns, and L. Novotny, Phys. Rev. Lett. 121, 033602 (2018).

[27] J. Ahn, Z. Xu, J. Bang, Y.-H. Deng, T. M. Hoang, Q. Han, R.-M. Ma, and T. Li, Phys. Rev. Lett. 121, 033603 (2018).

[28] M. Carlesso, M. Paternostro, H. Ulbricht, and A. Bassi, arXiv:1710.08695. 
[29] Y. Ma, T. M. Hoang, M. Gong, T. Li, and Z.-Q. Yin, Phys. Rev. A 96, 023827 (2017)

[30] S. Kuhn, B. A. Stickler, A. Kosloff, F. Patolsky, K. Hornberger, M. Arndt, and J. Millen, Nat. Commun. 8, 1670 (2017).

[31] B. A. Stickler, B. Papendell, S. Kuhn, J. Millen, M. Arndt, and K. Hornberger, arXiv:1803.01778.

[32] M. Carlesso, M. Paternostro, H. Ulbricht, A. Vinante, and A. Bassi, New J. Phys. 20, 083022 (2018).

[33] A. Manjavacas, F. J. Rodríguez-Fortuño, F. J. García de Abajo, and A. V. Zayats, Phys. Rev. Lett. 118, 133605 (2017).

[34] M. Rashid, M. Toroś, and H. Ulbricht, Quantum Meas. Quantum Metrol. 4, 17 (2018).

[35] M. Toroś, M. Rashid, and H. Ulbricht, Phys. Rev. A 98, 053803 (2018).

[36] M. Toroś, M. Rashid, and H. Ulbricht (to be published).

[37] See Supplementary Material at http://link.aps.org/ supplemental/10.1103/PhysRevLett.121.253601, which includes Refs. [38-41]. Supplementary Material contains information on the theoretical description of the system, the numerical simulations, as well as discussions on single-side modulation, torque sensitivity and limits of gas and photon damping.

[38] S. W. Ellingson, Radio Systems Engineering (Cambridge University Press, Cambridge, England, 2016).

[39] K. Ekinci, Y. Yang, and M. Roukes J. Appl. Phys. 95, 2682 (2004).

[40] K. Li, H. Fu, and Y. Li, Phys. Rev. A 98, 023862 (2018).

[41] C. Zhong and F. Robicheaux, Phys. Rev. A 95, 053421 (2017).

[42] P. H. Kim, C. Doolin, B. D. Hauer, A. J. MacDonald, M. R. Freeman, P. E. Barclay, and J. P. Davis, Appl. Phys. Lett. 102, 053102 (2013).

[43] D. Rugar, R. Budakian, H. J. Mamin, and B. W. Chui, Nature (London) 430, 329 (2004).

[44] P. Nagornykh, J. E. Coppock, J. P. J. Murphy, and B. E. Kane, Phys. Rev. B 96, 035402 (2017).

[45] J. F. Ralph, M. Toroš, S. Maskell, K. Jacobs, M. Rashid, A. J. Setter, and H. Ulbricht, Phys. Rev. A 98, 010102 (2018). [46] https://doi.org/10.5258/SOTON/D0523. 